\title{
In Silico Analysis of the Effects of Fibroblasts Coupling to Atrial Myocytes under Conditions of Atrial Fibrillation Remodeling
}

\author{
Jorge Sánchez, Beatriz Trénor, Javier Saiz \\ Centro de Investigación e Innovación en Bioingeniería, Universitat Politècnica de València, \\ Valencia, 46022, Spain
}

\begin{abstract}
Under chronic atrial fibrillation (cAF), cardiac tissue experiences electrophysiological and structural remodeling, including fibrosis. Fibrosis in the atrial tissue has an important impact on myocyte action potential (AP) and its propagation. The objective of this work is to explore fibloblasts characteristics and their impact on myocytes AP. Human atrial myocyte and fibroblast electrophysiology is simulated using mathematical models proposed by Koivumaki et al., including healthy and cAF remodeled myocytes. Cellular simulations were run using three different resting membrane potentials for the fibroblasts (RMPf) and different numbers of coupled fibroblasts to a single myocyte in $O D$ and with different fibroblast densities in 1D. Results from single cell simulations show that myocyte's AP duration (APD) depends on both RMPf and the number of coupled fibroblasts. In $1 D$ simulation, conduction velocity (CV) depends on RMPf and density along the strand. In conclusion, fibroblasts RMPf significantly alters myocyte AP and its propagation along cardiac tissue. Myocytes affected by cAF remodeling seem to be less sensitive to changes in fibroblasts RMPf.
\end{abstract}

\section{Introduction}

Atrial fibrillation (AF) is the most common cardiac arrhythmia. Under chronic atrial fibrillation (cAF) cardiac tissue experiences electrophysiological and structural remodeling. Fibroblasts density increases during AF and has an important role in the generation and maintenance of atrial reentries. Different studies suggest that fibroblasts present in the atrial substrate are electrically coupled to myocytes [1], [2]. Recent experimental studies have shown that fibroblasts express sodium channels allowing an inward current $\left(\mathrm{I}_{\mathrm{Na}}\right)$ and their coupling with myocytes alter the AP.[3-5].

Computational simulations of cellular electrophysiology gives the opportunity to study the role that fibroblasts play in the atrial substrate. It also gives the flexibility to modify cellular electrophysiology and vary different characteristics in order to analyze the impact of the electrical coupling with normal and electrical remodeled myocytes under cAF.

In this study, fibroblast sodium current will be updated using recent experimental data, RMPf will be varied to analyze its effects on the myocyte AP. This study also analyzes the different effects of fibroblast-myocyte coupling under normal and cAF conditions in structurally and electrophysiologically remodeled $1 \mathrm{D}$ atrial tissue.

\section{Materials and methods}

\subsection{Action potential models}

In this study we used human models of the atrial myocytes and fibroblasts from Koivumaki et al. [5], [6]. In the fibroblast model the sodium current was updated according to recent experimental results [4]. The electrical remodeling was introduced modifying ion channel conductances for L-type $\mathrm{Ca}^{2+}$ current $\left(\mathrm{I}_{\mathrm{CaL}}\right.$, transient outward $\mathrm{K}^{+}$current ( $\mathrm{I}_{\mathrm{to}}$, sustained outward $\mathrm{K}^{+}$ current $\left(\mathrm{I}_{\text {sus }}\right.$, time independent $\mathrm{K}^{+}$current $\left(\mathrm{I}_{\mathrm{K} 1)}\right.$, ryanodine receptors (RyR) and $\mathrm{Na}^{+} / \mathrm{Ca}^{2+}$ exchanger (NCX), sarcoplasmic reticulum $\mathrm{Ca}^{2+}$ ATPase (SERCA) pump and parameters of time constants for $\mathrm{I}_{\mathrm{CaL}}, \mathrm{I}_{\mathrm{to}}$ and $\mathrm{I}_{\mathrm{Na}}$, as described in Koivumaki et al.[7].

\subsection{Cellular simulations (0D)}

To compute the membrane potential in a cell equation (1) will be used:

$$
C m \frac{d V}{d t}+I_{\text {ion }}+\sum_{k=1}^{n} G_{\text {gap }}\left(V_{f}-V\right)=0
$$

Where $C_{m}$ is the membrane capacitance, $I_{i o n}$ is the ionic current flowing through ionic channels, $\mathrm{V}$ is the membrane potential in the myocyte, $\mathrm{n}$ is the number of attached fibroblasts to one myocyte and $\mathrm{G}_{\text {gap }}$ is the coupling conductance between myocyte and fibroblast. $\mathrm{G}_{\text {gap }}$ was set to $0.5 \mathrm{nS}$ [1]. Additionally, the number of attached fibroblasts to a single myocyte varied from 0 to 
9 and also the resting membrane potential of the fibroblast (RMPf) was set to different values $(-30,-45$, and $-65 \mathrm{mV}$ ) [8]. APDs were measured at $90 \%$ of repolarization $\left(\mathrm{APD}_{90}\right)$.

\subsection{Fiber simulations (1D)}

To simulate the electrical propagation of the action potential along a strand, the monodomain approximation diffusion-reaction equation (2) was used.

$$
\nabla(D \nabla \mathrm{V})=\mathrm{Cm} \frac{\mathrm{dV}}{\mathrm{dt}}+\text { Iion }(2)
$$

Where $\mathrm{D}$ is the diffusion coefficient, $\mathrm{C}_{\mathrm{m}}$ is the membrane capacitance and $\mathrm{I}_{\text {ion }}$ stands for ionic currents through the membrane. Fibroblasts were randomly distributed along the strand with two different densities (10\% and 20\%) and three different values for the RMPf ($30,-45$, and $-65 \mathrm{mV})$. Diffusion coefficient was calculated considering geometrical dimensions from the cell, gap junctions conductance, and intracellular medium conductance which was adjusted to achieve physiological conduction velocities (CV) along a strand of myocytes [9].

Depolarization upstroke was measured in a fibroblast near the centre of the strand and in one myocyte before and after the fibroblast to see the effect on the myocyte

depolarization upstroke as shown in Figure.

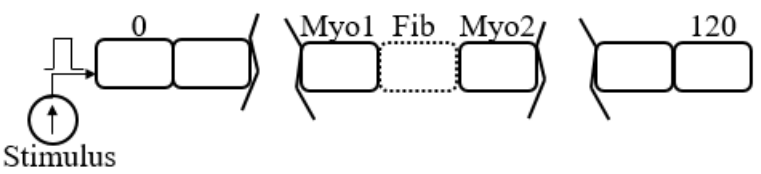

Figure 1. Strand configuration indicating the elements were measurements were taken (Myo1, Fib, and Myo2).

\subsection{Simulation protocols and numerical methods}

In $0 \mathrm{D}$ simulations the number of attached fibroblasts to a single myocyte was varied, as well as the value of RMPf. The applied stimuli had an amplitude of twice the threshold value. For 1D simulations, monodomain approach was solved using FDM with a space discretization of $100 \mu \mathrm{m}$ for myocytes and $10 \mu \mathrm{m}$ for fibroblasts and a time step of $10 \mu \mathrm{s}$. The first element was stimulated with an amplitude of twice its threshold. Both simulations reached steady state with a BCL of 1s before measurements were taken.

\section{Results and discussion}

Fibroblast coupling is expected to modulate myocyte AP characteristics. Results from 0D simulations are depicted in Figure 2, which shows how RMPf and the
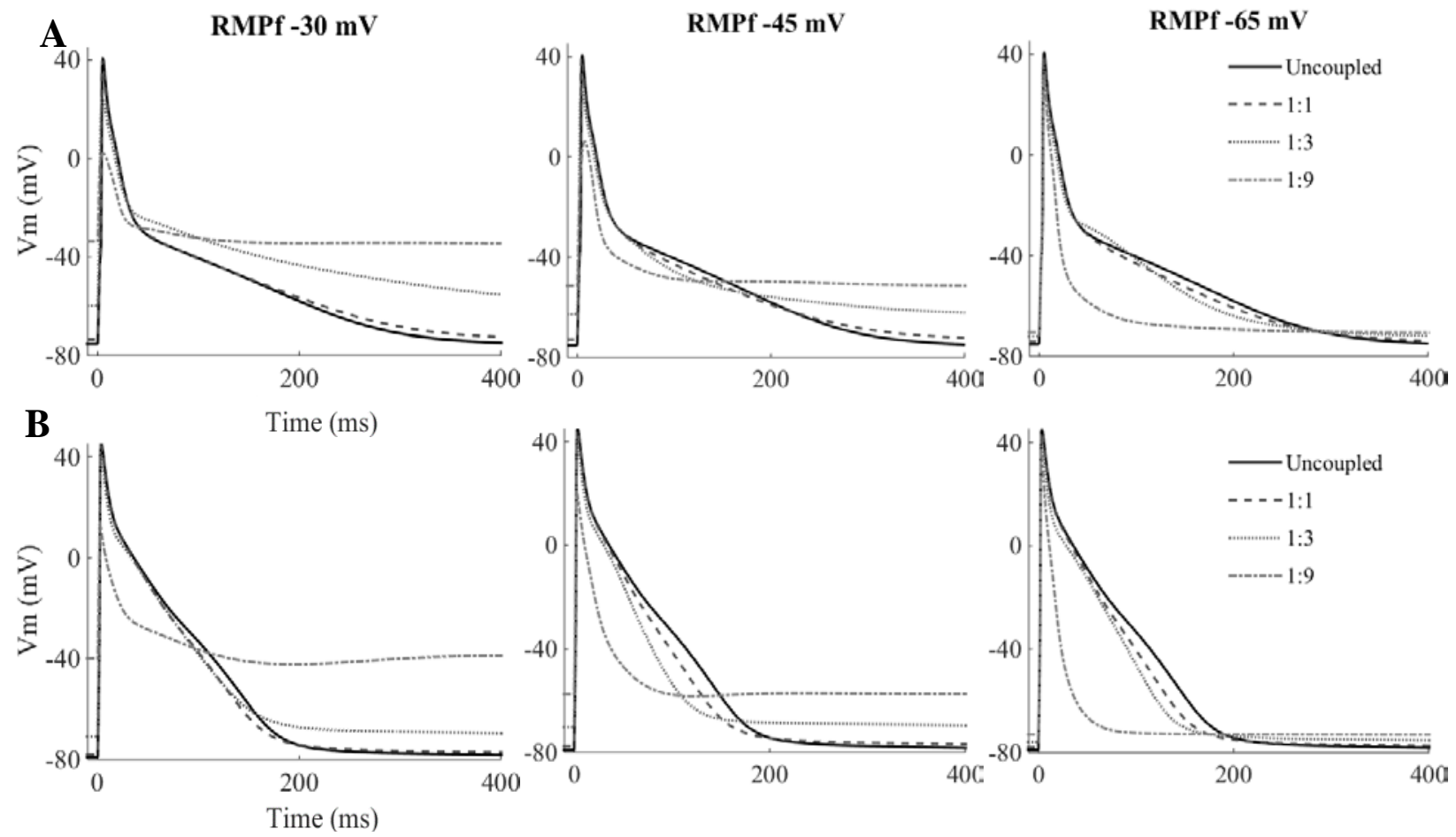

Figure 2. Simulations of isolated myocyte with a different number of attached fibroblasts and different RMPf, from left to right $-30,-45,-65 \mathrm{mV}$, respectively. Panel A represents APs for myocyte under normal conditions (nSR) and panel B APs for myocytes under cAF remodeling. 
number of coupled fibroblasts to a single myocyte have an influence on $\mathrm{APD}_{90}$ and resting membrane potential (RMP) of the myocyte in normal nSR conditions (panel A) and under cAF remodeling conditions (panel B).

Uncoupled myocyte $\mathrm{APD}_{90}$ is $233.2 \mathrm{~ms}$ and RMP is $74.8 \mathrm{mV}$ under $\mathrm{nSR}$ (control values). However, when coupled with 1 or 3 fibroblasts with RMPf of $-65 \mathrm{mV}$ (panel A right graph), $\mathrm{APD}_{90}$ is reduced to $91.68 \%$ and $78.04 \%$ of control and myocyte RMP is reduced to 99.18\% and $96.50 \%$ of control. When RMPf is $-45 \mathrm{mV}$ (panel A middle graph), APD 90 is reduced to $94.43 \%$ and $70.20 \%$ of control value and the myocyte RMP is $97.36 \%$ and $83.98 \%$ of control. In both cases myocyte $\mathrm{APD}_{90}$ is shortenned and myocyte RMP is more depolarized when RMPf is less negative and coupled to 3 fibroblasts. Additionally, when RMPf is $-30 \mathrm{mV}$ (panel A left graph) and coupled to 1 or 3 fibroblasts, myocyte $\mathrm{APD}_{90}$ was longer (102\% and 137\%, respectively) and their RMP is 98.15\% and $80.12 \%$ of control. When 9 fibroblasts are coupled $\mathrm{APD}_{90}$ is reduced to $30 \%$ of control approximately with all RMPf values, also myocyte RMP is significantly depolarized when RMPf is less negative.

Under cAF remodeling conditions (panel B), uncoupled myocyte $\mathrm{APD}_{90}$ is $168 \mathrm{~ms}$ and RMP is -79.04 $\mathrm{mV}$ (control values) due to electrical remodeling. For 1 or 3 fibroblasts coupled to a single myocyte and RMPf -65 $\mathrm{mV}$ (panel B right graph), APD90 is $90 \%$ and myocytes RMP $98.78 \%$ of control. However when coupled with 1 or 3 fibroblasts with RMPf of $-45 \mathrm{mV}$ (panel B middle graph), APD $_{90}$ is $86.82 \%$ and $66.75 \%$ of control with myocyte RMP of $98.14 \%$ and $88.86 \%$ of control. It is important to highlight that when coupled to 1 or 3 fibroblasts and RMPf of $-30 \mathrm{mV}$ (panel B left graph), all $\mathrm{APD}_{90}$ are shortenned. Furthermore, coupling to 9 fibroblasts results in an $\mathrm{APD}_{90}$ and myocytes RMP reduction of $50 \%$ of control. Electrically remodeled

A RMPf $-30 \mathrm{mV}$
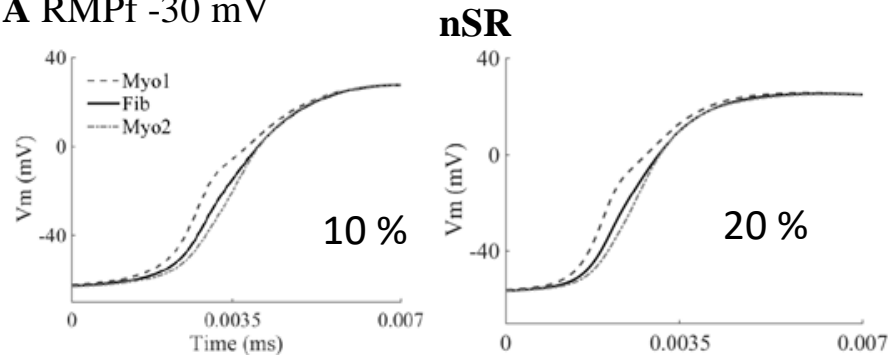

B RMPf $-45 \mathrm{mV}$
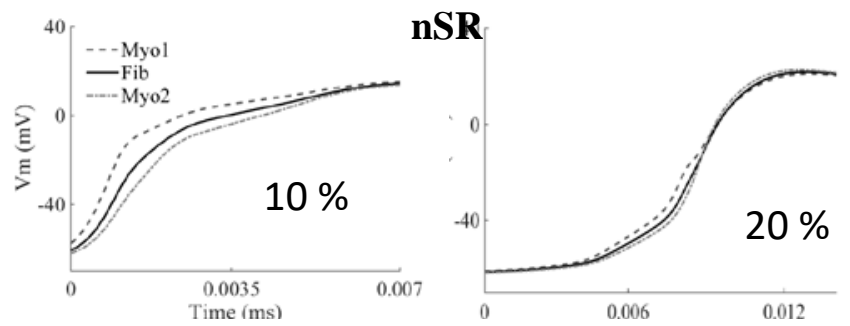

myocytes are less sensitive to the effect of fibroblast coupling and the variation of the RMPf.

As observed in OD simulations, fibroblasts coupling has an important effect on myocytes AP. In 1D simulations, $\mathrm{CV}$ was analyzed to study fibroblasts characteristics in a tissue strand. $\mathrm{CV}$ in physiological conditions was $70 \mathrm{~cm} / \mathrm{s}$ and in cAF CV was reduced to $68.6 \mathrm{~cm} / \mathrm{s}$.

Error! Reference source not found. shows the influence of fibroblast coupling on the upstroke of the AP. Values obtained for $\mathrm{dV} / \mathrm{dt}_{\max }$ during the depolarization upstroke of myocytes were $103 \mathrm{mV} / \mathrm{s}$ and $100 \mathrm{mV} / \mathrm{s}$ in $\mathrm{nSR}$ and cAF, respectively (control values) in the absence of fibrosis.

In $\mathrm{nSR} \mathrm{dV} / \mathrm{dt}_{\max }$ of myocytes located before the fibroblast (Myo1 in Figure 1) are reduced depending on the RMPf and the fibrosis density in the strand. For $10 \%$ density and RMPf of $-30 \mathrm{mV}$ (panel A, left graph) $\mathrm{dV} / \mathrm{dt}_{\max }$ is reduced to $57 \%$ of control value while for the myocyte after the fibroblasts (Myo2 in Figure 1) $\mathrm{dV} / \mathrm{dt}_{\max }$ is $47 \%$ of control. With RMPf of $-45 \mathrm{mV}$ (panel C, left graph) $\mathrm{dV} / \mathrm{dt}_{\max }$ is $65 \%$ of control for Myo1 while for Myo2 the value is $53 \%$ of control. When fibroblast density is increased to $20 \% \mathrm{dV} / \mathrm{dt}_{\max }$ with $-30 \mathrm{mV}$ (panel A, second graph) in Myo1 is 60\% and in Myo2 is 37\% of control, and with RMPf of $-45 \mathrm{mV}$ (panel B, second graph) the first myocyte presents a $\mathrm{dV} / \mathrm{dt}_{\max }$ of $25 \%$ and the second myocyte $34 \%$ of control.

$\mathrm{CV}$ is also reduced with increasing fibroblast density along the strand and depending on RMPf. Results from simulations in nSR shows that with densities of $10 \%$ with all three RMPf, CV is reduced (to $87 \%$, 85\%, and 63\% of control value without fibrosis, respectively). When fibrosis density increases to $20 \% \mathrm{CV}$ does not follow the same reduction trend. While with $-30 \mathrm{mV} R M P f, C V$ is reduced to $63 \%$ of control value, with $-45 \mathrm{mV}$ and -65

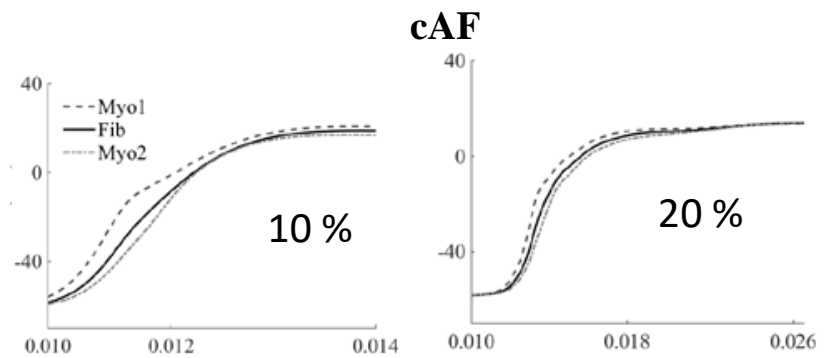

Figure 1. Depolarization upstroke for Myo 1, Fibroblast and Myo2 in 1D simulated strand for different conditions. Panel A) $10 \%$ and 20\% fibrosis density with a RMPf of $-30 \mathrm{mV}$ and nSR. Panel B) 10\% and 20\% fibrosis density with a RMPf of $30 \mathrm{mV}$ and cAF. Panel C) 10\% and 20\% density with RMPf of $-45 \mathrm{mV}$ and nSR. Panel D) 10\% and 20\% fibrosis density with a RMPf of $-45 \mathrm{mV}$ and $\mathrm{cAF}$. 
$\mathrm{mV} \mathrm{CV}$ is only reduced to $74 \%$ and $75 \%$ of control value.

When electrical remodeling is introduced to simulate cAF conditions, values of $\mathrm{dV} / \mathrm{dt}_{\max }$ in the upstroke of the depolarization phase are also affected. For density of $10 \%$ and RMPf of $-30 \mathrm{mV}$ (panel B left graph), $\mathrm{dV} / \mathrm{dt}_{\max }$ for Myo1 is $66 \%$ of control and with RMPf of $-45 \mathrm{mV}$ (panel D left graph) is $43 \%$ of control, values for Myo2 are $51 \%$ and $28 \%$ of control respectively. When the density of fibroblasts is $20 \%$ and RMPf of $-30 \mathrm{mV}$ for Myo1 (panel B second graph) $\mathrm{dV} / \mathrm{dt}_{\max }$ is $58 \%$ and with a RMPf of -45 $\mathrm{mV}$ for Myo1 (panel D second graph) 54\% of control, for

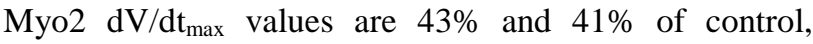
respectively.

$\mathrm{CV}$ in $\mathrm{CAF}$ for $10 \%$ density and with all three RMPf shows the same percentage of reduction as in nSR. When fibroblast density increases, $\mathrm{CV}$ decreases as RMPf is depolarized (74\%, 71\%, and $67 \%$ of control, respectively). Additionally, RMP of the myocyte is not significantly affected.

Our results in $0 \mathrm{D}$ and $1 \mathrm{D}$ show that fibroblasts play an important role in both physiological nSR and in $\mathrm{cAF}$ conditions. They modify atrial substrate, which defines tissue properties for the action potential to propagate. Results obtained are in accordance with experimental results that show a decay in $\mathrm{CV}$ when density of fibroblast increases [10], [11]. It is interesting to notice that during CAF, CV seems to be less sensitive to present variability with RMPf.

\section{Conclusion}

In conclusion, fibroblasts effects on the myocyte AP depends on the value of their RMPf and the number of fibroblasts coupled to a myocyte as observed in $0 \mathrm{D}$ simulations. In 1D, fibroblast density and RMPf alters the action potential depolarization upstroke which modifies the CV along a tissue strand. Fibroblasts and their RMPf significantly alter myocyte AP and its propagation along cardiac tissue. Myocytes affected by cAF remodeling seem to be less sensitive to changes in fibroblasts RMPf.

\section{Acknowledgment}

This work was partially supported by the "Plan Estatal de Investigación Científica y Técnica y de Innovación 2013-2016” from the Ministerio de Economía, Industria y Competitividad of Spain (DPI2016-75799-R) and AEI/FEDER, UE, and by the "Programa Prometeu (PROMETEU/2016/088) de la Conselleria d'Educació, Formació I Ocupació, Generalitat Valenciana”.

\section{References}

[1] V. Jacquemet and C. S. Henriquez, "Modeling cardiac fibroblast, their interactions with myocytes aand their Impact on Impulse Propagation,” Eruopace, vol. 9, no. Suppl 6, pp. 1-19, 2013.

[2] K. S. McDowell et al., "Mechanistic inquiry into the role of tissue remodeling in fibrotic lesions in human atrial fibrillation," Biophys. J., vol. 104, no. 12, pp. 2764-2773, 2013.

[3] A. Chatelier et al., "A distinct de novo expression of $\mathrm{Na}$ v 1.5 sodium channels in human atrial fibroblasts differentiated into myofibroblasts," J. Physiol., vol. 590, no. 17, pp. 4307-4319, 2012.

[4] C. Poulet, S. Künzel, E. Büttner, D. Lindner, D. Westermann, and U. Ravens, "Altered physiological functions and ion currents in atrial fibroblasts from patients with chronic atrial fibrillation.," Physiol. Rep., vol. 4, no. 2, p. e12681, 2016.

[5] J. Koivumäki et al., "Na+ current expression in human atrial myofibroblasts: Identity and functional roles," Front. Physiol., vol. 5 JUL, no. August, pp. 1-14, 2014.

[6] J. T. Koivumäki, T. Korhonen, and P. Tavi, "Impact of sarcoplasmic reticulum calcium release on calcium dynamics and action potential morphology in human atrial myocytes: A computational study," PLoS Comput. Biol., vol. 7, no. 1, 2011.

[7] J. T. Koivumäki, G. Seemann, M. M. Maleckar, and P. Tavi, "In Silico Screening of the Key Cellular Remodeling Targets in Chronic Atrial Fibrillation," PLoS Comput. Biol., vol. 10, no. 5, 2014.

[8] M. M. Maleckar, J. L. Greenstein, W. R. Giles, and N. A. Trayanova, "Electrotonic coupling between human atrial myocytes and fibroblasts alters myocyte excitability and repolarization,” Biophys. J., vol. 97, no. 8, pp. 2179-2190, 2009.

[9] David M. Harrild, Craig S. Hen, “A Computer Model of Normal Conduction in the Human Atria," Circ. Res., vol. 87, no. 7, pp. e25-e36, 2000.

[10] M. Miragoli, G. Gaudesius, and S. Rohr, "Electrotonic modulation of cardiac impulse conduction by myofibroblasts," Circ. Res., vol. 98, no. 6, pp. 801810, 2006.

[11] F. Jousset, A. Maguy, S. Rohr, and J. P. Kucera, "Myofibroblasts electrotonically coupled to cardiomyocytes alter conduction: Insights at the cellular level from a detailed in silico tissue structure model," Front. Physiol., vol. 7, no. OCT, pp. 1-23, 2016.

Jorge Sánchez Arciniegas

Centro de Investigación e Innovación en Bioingeniería, Universitat Politècnica de València, Valencia, 46022, Spain

E-mail: jorsana4@posgrado.upv.es 